Brit. Heart J., 1965, 27, 926.

\title{
ORAL TREATMENT OF A-V BLOCK AND OTHER BRADYCARDIAS WITH SUSTAINED ACTION ISOPRENALINE
}

\author{
BY \\ NIS I. NISSEN AND ÅGE CHR. THOMSEN \\ From Sundby Hospital, Medical Department, Bispebjerg Hospital, Medical Department B and \\ Blegdamshospitalet, Medical Department, Copenhagen, Denmark \\ Received March 5, 1965
}

Bradycardia can be due to disturbances in the cardiac conduction system, of which the most common is the atrio-ventricular (a-v) block. Medical treatment of this disorder usually consists in blocking the vagus-inhibitory effect upon the heart or in stimulation, by administering sympathomimetic substances. During recent years, interest has been focused on the use of sympathomimetic amines, especially isoprenaline hydrochloride (Nathanson and Miller, 1949, 1952). In a normal heart this agent, which predominantly acts on the $\beta$-receptors (Moran, 1963), will increase the heart rate by stimulating the sinoatrial and a-v node, while in the presence of a complete a-v block it accelerates the impulse centres in the ventricles. It has less tendency than adrenaline to stimulate ventricular ectopic foci.

The hæmodynamic effect of this drug has been studied, among others, by Levinson et al. (1959) who during cardiac catheterization injected isoprenaline into the pulmonary arteries of patients with complete a-v block and bradycardia. The ventricular rate and cardiac output rose, while the stroke volume remained unchanged. As a manifestation of the vasodilator effect of isoprenaline, a decrease in peripheral resistance, in the pulmonary as well as in the systemic circulation, was noted.

A major drawback of oral treatment with isoprenaline hydrochloride, however, was the shortlasting effect (about half an hour) that necessitated administration every hour or half hour. It was an advance, therefore, when Robbin and Dack (1959) introduced a tablet with sustained release of the active substance. Treating 15 patients with a-v block, they obtained a favourable result in 13 . Reversion to sinus rhythm was observed in 2 patients, but was stable in only one. In about onethird of the patients the ventricular rate increased. Also, in the remaining patients the Stokes-Adams attacks ceased, though the pulse rate was not affected. In 2 patients the treatment had to be discontinued because of side-effects in the form of palpitations and muscular tremor (Dack and Robbin, 1961). Fleming and Mirams (1963) treated 8 patients with heart block. In all, the heart rate increased and the Stokes-Adams attacks ceased. Four reverted to sinus rhythm, but one only temporarily. Side-effects in the form of slight tremor were observed in 2 patients, and one developed ventricular extrasystoles which disappeared when the dosage was reduced.

As the general experience with sustained action isoprenaline is still very limited, we believe it is of interest to publish our results from the treatment of a-v block and other types of bradycardia with this agent.

\section{SubJECTS AND METHODS}

S.A.I.H. The preparation used (saventrine) is made, according to information from the makers, by coating multiple small cores of inert material with isoprenaline hydrochloride and enclosing each granule in a varying number of separate layers of ethylcellulose. In the gastro-intestinal tract this substance will 
dissolve independently of individual variations in $p \mathrm{H}$ and enzyme activity, but proportionately to time. The time of release of the active medication is directly proportional to the number of layers of ethylcellulose surrounding an individual granule. It is possible, therefore, to make tablets with a constant release of isoprenaline hydrochloride through several hours, as far as the present tablets are concerned allegedly over 8 hours ("Sustained action isoprenaline hydrochloride", S.A.I.H.).

Dosage. S.A.I.H. was administered in the form of tablets of $30 \mathrm{mg}$. The initial dose was 1 tablet 3 or 4 times daily. Thereafter, the dose was increased by 1 tablet daily. The maximum dose was 7 tablets 4 times daily (840 mg.). In a few cases, we preferred dividing the daily dose further, administering the drug every other or third hour during the daytime, as this appeared to reduce the tendency to palpitations. The maintenance dose had to be fixed individually, depending upon the objective effect and side-effects. The patients were told to swallow the tablets whole.

Patients. The subjects (Table I) comprised 32 consecutive patients having Stokes-Adams attacks and/or bradycardia (pulse rate below 50 a minute). Twenty-eight patients had atrio-ventricular block, which was a third degree block (complete) in 23 , a second degree block $(2: 1$ or $3: 1)$ in 3 , a first degree block with prolonged $\mathrm{P}-\mathrm{Q}$ in one. Of the remaining 5 patients, 2 had nodal rhythm, 2 had atrial fibrillation, and one had sinoatrial block.

Stokes-Adams attacks occurred on one or more occasions in 18 patients. In most of the patients the xtiology was unknown, but it was presumably arteriosclerosis. Many of the patients had previously been treated with ephedrine, amphetamine, or atropine without major effect (cf. also Table I). Oral diuretics had usually been administered to patients with cardiac decompensation, and this treatment was continued.

The cardiogram, blood pressure, and heart rate were recorded daily for the in-patients, and weekly for the out-patients. The pulse rate was counted morning, noon, and night during the stay in hospital, and after discharge by the patients themselves.

\section{Results}

The therapeutic results are presented in Table II.

Some increase in the pulse rate was observed in most of the patients, but only 18 showed an increase by at least one-third of the initial value. The final values stated in this Table indicate the pulse rate on a maintenance dose. On the maximum dose higher values were recorded in several cases.

During the treatment with S.A.I.H. none of the patients had Stokes-Adams attacks. Nine had previously had such frequent attacks that the effect of the treatment was beyond doubt. In the other 9 patients the attacks occurred at such long intervals that, owing to the relatively short observation period, it was not possible to evaluate the result.

The treatment induced altered rhythm in 6 patients. In 2 (Cases 19 and 30) sinus rhythm was restored. Complete block was transformed to a first-degree block in one (Case 8), into a seconddegree block in one patient (Case 20), and into atrial fibrillation in one instance (Case 9). In one patient (Case 32), who had an unstable rhythm alternating between complete and 3:1 block, a consistent complete block was the final result.

In 6 out of 12 patients with manifest signs of heart failure there was clear improvement. In the others the dyspnœa at rest decreased. In 3 disabled patients it was possible for former physical activity and work to be resumed. An improvement in the general condition shown by increased energy and mobility, less fatigue and dizziness, and decrease in need for sleep was a commonly observed feature in the 22 patients who completed the treatment.

The medication was discontinued in 12 patients. In 6 patients this was due to insufficient effect on the heart rate (Cases 12 (cf. case history), 13, 16, 23, 25, and 27). In 4 patients (Cases 11, 14, 15, and 18) the mental state made co-operation impossible. The changes in rhythm in Cases 9 and 29, mentioned above, made cessation of treatment possible.

Side-effects. Some of the patients experienced difficulty in falling asleep if the last dose was administered immediately before bedtime, and many complained of palpitations. Both symptoms disappeared after suitable alterations in the administration of the drug or reduction of the dosage. A few patients temporarily had an uncharacteristic feeling of tremor without objective signs. More 
TABLE I

Case Material

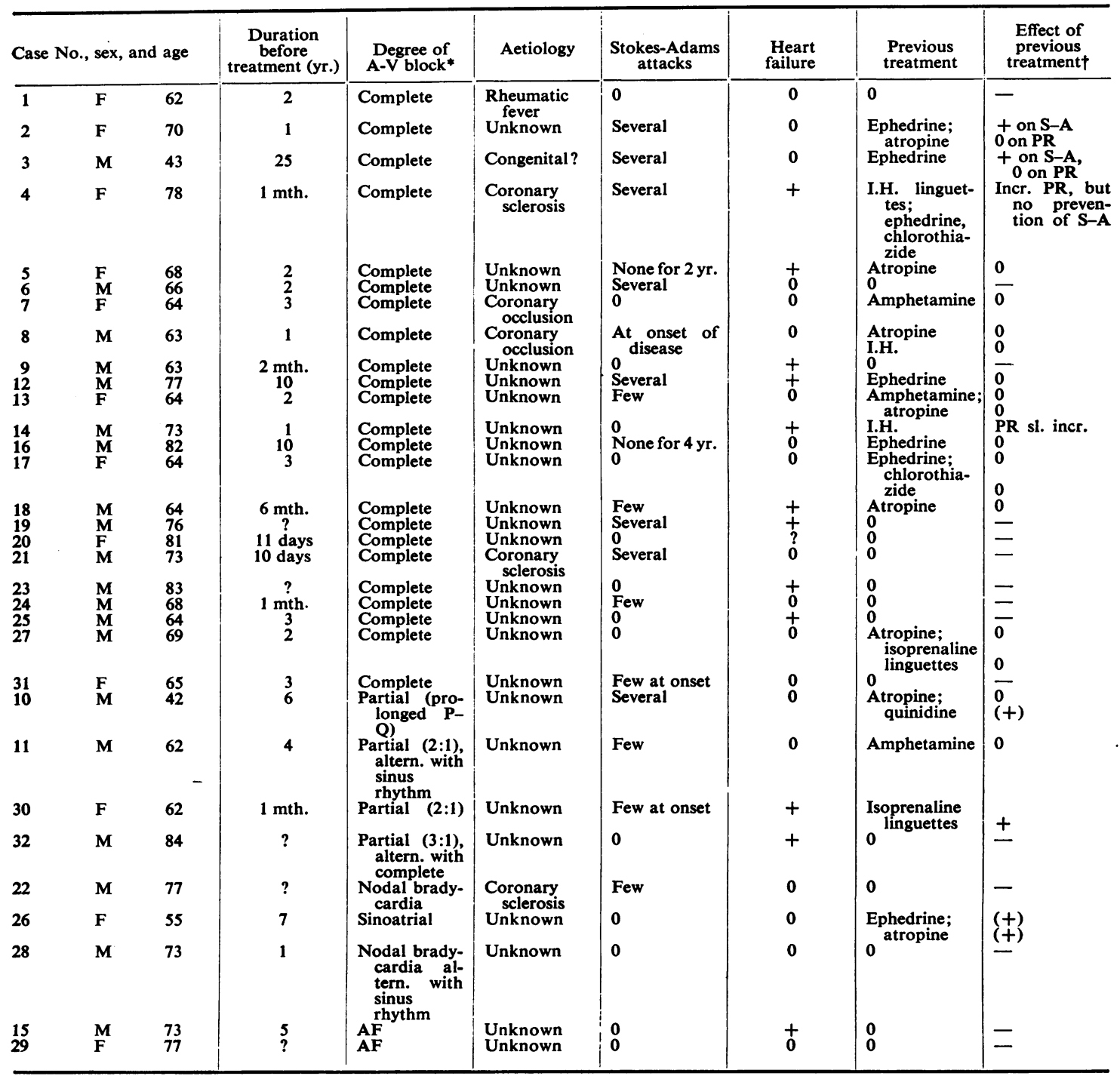

*AF is atrial fibrillation.

T $P R$ is pulse rate; $S-A$ is Stokes-Adams attacks.

frequent extrasystoles were observed in 3 patients (Cases 6, 7, and 23), but they disappeared during continued medication.

The changes in blood pressure are shown in Table II. In the majority of patients a slight fall in systolic as well as in diastolic pressure was noted. In general, the drug was well tolerated, and no patient showed gastro-intestinal disturbances that could be ascribed to the treatment.

One patient (Case 1) developed coronary occlusion during the treatment; however, she was suffering from essential hypercholesterolæmia and 2 years previously had had a cerebrovascular attack. 
TABLE II

Results from Treatment of Heart Block with Sustained Action Isoprenaline Hydrochloride

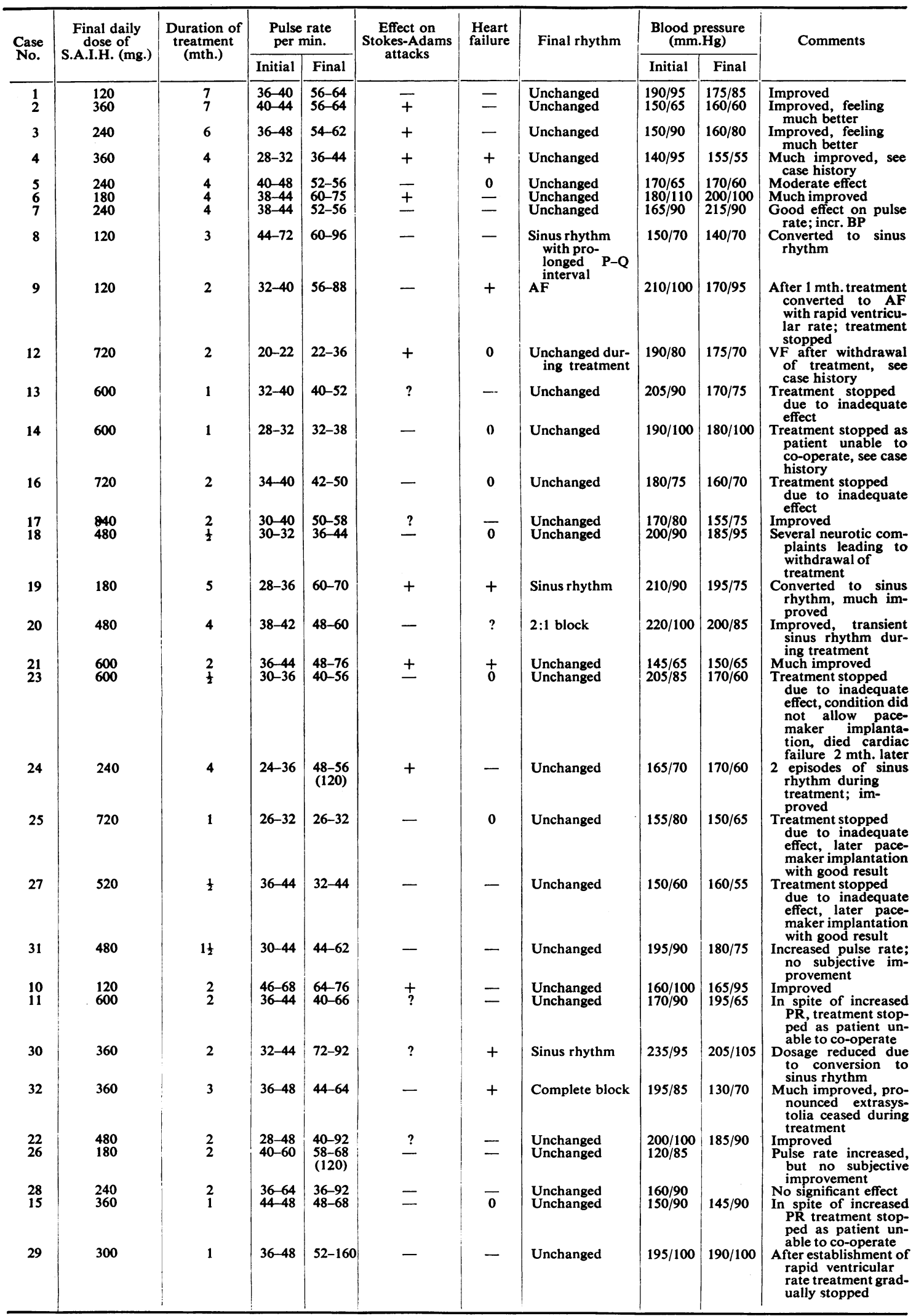


Consequently, the coronary occlusion was not interpreted as a complication of the treatment which was continued with favourable results.

A few characteristic case histories are reported below.

\section{Case Histories}

Case 4. A woman, aged 78, with an acute onset of complete atrio-ventricular block, presumably due to arteriosclerosis, was admitted to a department of thoracic surgery for implantation of an internal pacemaker. This plan was given up because of the poor general and cardiac condition. Immediately after transfer to the medical department the patient had several Stokes-Adams attacks. During treatment with S.A.I.H., 90 $\mathrm{mg}$. daily, increasing to $600 \mathrm{mg}$. daily, there was a significant increase in the pulse rate, improvement in the cardiac condition, and the Stokes-Adams attacks ceased. She was feeling well when discharged on a maintenance dose of $360 \mathrm{mg}$ daily.

Case 6. A 66-year-old man had a two-year history of atrio-ventricular block of unknown ætiology, which involved numerous Stokes-Adams attacks, disabling fatigue, and severe anxiety neurosis. On S.A.I.H., $90 \mathrm{mg}$. daily, increasing to a maximum of $\mathbf{3 0 0} \mathrm{mg}$. daily, the Stokes-Adams attacks ceased, and there was a pronounced subjective improvement. Attempts at withdrawing the medication immediately resulted in a relapse. The condition is now (5th month) stable on a maintenance dose of $120 \mathrm{mg}$. daily.

Case 8. A man, aged 63, had a year's history of complete atrio-ventricular block following coronary occlusion. Initially, he had a few Stokes-Adams attacks, later only abortive attacks. On S.A.I.H. therapy these disappeared, and there was an obvious subjective improvement. The complete a-v block reverted after 4-5 days treatment to sinus rhythm with a prolonged $P-Q$ interval. This condition is now stable on continued medication (5th month).

Case 9. This 63-year-old man had a two-year history of atrial fibrillation, and for two months bradycardia with increasing heart failure. The cardiogram revealed atrial fibrillation with ventricular autonomy (pulse rate 32-40). After one month on S.A.I.H., the slow regular ventricular rhythm was changed to rapid irregular tachycardia with a rate of 100 to 150 . The tachycardia persisted after withdrawal of S.A.I.H., and digitalization was required to lower the heart rate to its present level of about 80 a minute.

Case 12. A 77-year-old man with a ten-year history of complete a-v block of unknown ætiology, was admitted because of frequent Stokes-Adams attacks and extreme bradycardia (20-22). During treatment with S.A.I.H. in doses up to $720 \mathrm{mg}$. daily there was only a negligible increase in the pulse rate, but the StokesAdams attacks ceased. Owing to the slight effect upon the heart rate, the implantation of a pacemaker was contemplated, and S.A.I.H. was gradually withdrawn. Immediately after the medication had been stopped, the patient on one day had numerous Stokes-Adams attacks, culminating in syncope with ventricular fibrillation. After treatment with external cardiac massage, defibrillation, and infusion of metaraminol, regular ventricular rhythm with a frequency of 20-30 was re-established. The cardiogram showed, as previously, complete a-v block. The patient was transferred to a department of thoracic surgery, where he was treated with a catheter electrode and later with implantation of a pacemaker. He was feeling well on discharge.

Case 14. A man, aged 73, with a one-year history of complete a-v block of unknown ætiology, was admitted because of bradycardia with cardiac failure. Only a slight increase in the pulse rate on S.A.I.H. in doses up to $600 \mathrm{mg}$. daily was observed. The maximum dosage was administered for only one week due to the patient's mental instability and lack of ability to co-operate. Following discontinuation of the treatment a pacemaker was implanted with a favourable result.

\section{Discussion}

As is evident from our investigations, complete a-v block may be effectively treated with S.A.I.H. in at least half the cases. Thus, our experience is largely in keeping with previous results reported by Dack and Robbin (1961) and Fleming and Mirams (1963). Unlike the latter authors, however, we found that a conversion to sinus rhythm was uncommon.

During recent years the use of pacemaker implantation in cases of complete heart block and Stokes-Adams attacks has increased. As emphasized by Fleming and Mirams (1963) the favourable results of medical therapy with S.A.I.H. may be expected to reduce the need for implantation of permanent internal pacemakers. However, the experience with both these methods is relatively limited and long-term results are not yet available. Until further evidence has accumulated con- 
cerning which of the methods is to be chosen in an individual case, the following treatment may be recommended.

Emergency cases of the Stokes-Adams syndrome with frequent syncopal episodes are given an initial intravenous infusion of isoprenaline (2-10 mg. per $1000 \mathrm{ml}$. glucose $5 \%$ ). If available, an external pacemaker should be kept ready for immediate use in case of ventricular asystole.

If the patient responds to the intravenous treatment, oral administration of S.A.I.H. is started and the intravenous treatment is gradually reduced. Maintenance dose of S.A.I.H. is adjusted as described above.

If the acutely ill patient does not respond to intravenous isoprenaline, electric pacing of the heart is indicated, preferably by a catheter electrode inserted into the right ventricle. Later a permanent internal pacemaker may be implanted.

When a-v block is caused by acute myocardial infarction or myocarditis, corticosteroid therapy can be valuable.

In less acute cases with no or infrequent Stokes-Adams attacks the treatment is started and maintained with S.A.I.H. If the ventricular rate remains low despite adequate dosage, implantation of pacemaker must be considered.

As we have shown, bradycardia due to other causes than chronic or recurrent complete a-v block may also afford an indication for S.A.I.H. The treatment is given in accordance with the principles described above.

\section{SUMMARY}

Thirty-two consecutive patients with atrio-ventricular block and bradycardia from other causes were treated with sustained-action isoprenaline hydrochloride (saventrine). A conspicuous effect upon Stokes-Adams attacks and heart rate was observed in more than half of the patients. The 22 patients who completed the treatment experienced considerable subjective improvement.

The principles of treatment of the Stokes-Adams syndrome and bradycardia are discussed and a scheme for the management of acute and chronic cases is proposed.

\section{REFERENCES}

Dack, S., and Robbin, S. R. (1961). Treatment of heart block and Adams-Stokes syndrome with sustained-action isoproterenol. J. Amer. med. Ass., 176, 505.

Fleming, H. A., and Mirams, J. A. (1963). A clinical trial of a sustained-action preparation of isoprenaline in the treatment of heart block. Lancet, 2, 214.

Levinson, D. C., Shubin, H., Gunther, L., and Meehan, J. P. (1959). Hemodynamic findings in heart block with slow ventricular rates, Amer.J. Cardiol., 4, 440.

Moran, N. C. (1963). Adrenergic receptors within the cardiovascular system. Circulation, $28,987$.

Nathanson, M. H., and Miller, H. (1949). Effect of 1-(3,4,-dihydroxyphenyl)-2-isopropylaminoethanol, (isopropylepinephrine) on the rhythmic property of the human heart. Proc. Soc. exp. Biol. (N.Y.), 70, 633.

- and - (1952). The action of norepinephrine, epinephrine and isopropyl norepinephrine on the rhythmic function of the heart. Circulation, 6, 238.

Robbin, S. R., and Dack, S. (1959). Treatment of Stokes-Adams attacks in heart block, with special reference to parenteral, sublingual and long-acting isoproterenol. Circulation, 20, 757. 